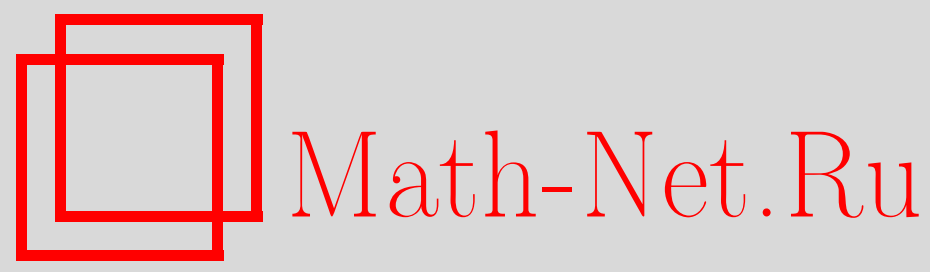

Н. И. Санду, О длине нижнего центрального ряда (производного ряда) коммутативной лупы Муфанг, Матем. заметки, 1997, том 62, выпуск 3, 475-478

DOI: https://doi.org/10.4213/mzm1629

Использование Общероссийского математического портала Math-Net.Ru подразумевает, что вы прочитали и согласны с пользовательским соглашением http://www.mathnet.ru/rus/agreement

Параметры загрузки:

IP : 3.85 .73 .92

26 апреля 2023 г., 14:09:10

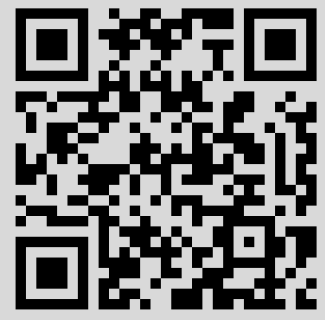




\section{О ДЛИНЕ НИЖНЕГО ЦЕНТРАЛЬНОГО РЯДА (ПРОИЗВОДНОГО РЯДА) КОММУТАТИВНОЙ ЛУПЫ МУФАНГ}

\section{Н. И. Санду}

В теории коммутативных луп Муфанг (КЛМ) хорошо известна замечательная теорема Брака-Слэби, играющая ключевую роль в исследовании таких луп: КЛМ с $n$ $(n \geqslant 2$ конечно) образующими центрально нильпотентна ступени не больше чем $n-1[1]$. Это означает, что длина нижнего центрального ряда таких луп не превосходит $n-1$. В [2], [3] построены примеры КЛМ с $n$ образующими, которые являются центрально нильпотентньми ступени $n-1$. Отсюда следует, что длина ступени нижнего центрального ряда свободной КЛМ с $n$ свободными образующими равна $n-1$. В [3] показано, что длина производного ряда свободной КЛМ с $n$ свободньги образующими равна $\min \left\{k \mid 3^{k} \geqslant 2 n-1\right\}$. Отметим также, что в [1]-[3] построены КЛМ, длина нижнего центрального ряда которых равна $\omega$. В данной заметке по аналогии с теорией групп [4] строятся КЛМ, длина нижнего центрального ряда (производного ряда) которых равна произвольному наперед заданному трансфинитному числу.

Теперь напомним некоторые понятия из теории КЛМ [1]. Коммутативная лупа Муфанг характеризуется тождеством

$$
x^{2} \cdot y z=x y \cdot x z
$$

Acсоииатор $[a, b, c]$ элементов $a, b, c \operatorname{KJM}(Q, \cdot) \equiv Q$ определяется равенством $a b \cdot c=$ $a[a, b, c] \cdot b c$. A cсочиант $Q^{\prime}$ КЛМ $Q$ - эта подгруппа, порожденная всеми ассоциаторами КЛМ $Q$. Она нормальна в $Q$. Нижний (трансфинитный) иентральный ряд КЛМ $Q$ имеет вид

$$
Q=Q_{0} \supseteq Q_{1} \supseteq \cdots \supseteq Q_{\omega} \supseteq Q_{\omega+1} \supseteq \cdots,
$$

где нормальная подлупа $Q_{\alpha+1}$ порождается всеми ассоциаторами вида $[a, u, v], a \in Q_{\alpha}$, $u, v \in Q$, а $Q_{\alpha}$ для предельного $\alpha$ есть пересечение всех предыдущих подлуп. Лупа $Q$ называется ZD-лупой, если ее нижний центральный ряд достигает единицы на каком-нибудь, быть может, трансфинитном месте. Наименьшее трансфинитное число $\alpha$, для которого $Q_{\alpha}=1$, называется ступенью ZD-лупы. (Отметим, что в [1] название для таких луп иное.) Если $\alpha$ - конечное число, то КЛМ $Q$ назьвается иентрально нильпотентной.

Аналогично, система нормальных подлуп

$$
Q=Q^{(0)} \supseteq Q^{(1)} \supseteq \cdots \supseteq Q^{(\omega)} \supseteq Q^{(\omega+1)} \supseteq \cdots
$$

будет (трансфинитным ) производным рядом КЛМ $Q$, если $Q^{(\alpha+1)}$ есть ассоциант подлупы $Q^{(\alpha)}$, а для предельного $\alpha$ подлупа $Q^{(\alpha)}$ есть пересечение предыдуших членов. КЛМ $Q$ назьвается RK-лупой ступени $\alpha$, если $Q^{(\alpha)}=1, Q^{(\beta)} \neq 1, \beta<\alpha$. Если $\alpha$ конечно, то КЛМ $Q$ назьвается иентрально разрешимой.

В дальнейшем под алгеброй будем понимать ассоциативную алгебру над полем из трех элементов. Пусть алгебра $A$ антикоммутативна, т.е. квадрат любого ее элемента равен нулю. В таком случае для любых $x, y \in A$ выполнено соотношение $x y=-y x$. 
Пусть $x_{1}, y_{1}, x_{2}, y_{2} \in A$. Положим

$$
x=\left(\begin{array}{l}
x_{1} \\
x_{2}
\end{array}\right), \quad y=\left(\begin{array}{l}
y_{1} \\
y_{2}
\end{array}\right)
$$

Используя указанные вьше свойства антикоммутативной алгебры, несложно проверить (см. также [3]), что операция '.':

$$
x \cdot y=\left(\begin{array}{c}
x_{1}+y_{1}+x_{1} y_{1} x_{2}+x_{1} y_{2} y_{1} \\
x_{2}+y_{2}+x_{2} y_{2} y_{1}+x_{2} y_{1} y_{2}
\end{array}\right)
$$

превращает $A \times A=Q$ в КЛМ (для этого необходимо убедиться в справедливости тождества (1)), которую назовем соответствующей алгебре А. Проверяется также, что

$$
[x, y, z]=\left(\begin{array}{l}
x_{1} y_{1} z_{2}+y_{1} z_{1} x_{2}+z_{1} x_{1} y_{2} \\
x_{2} y_{2} z_{1}+y_{2} z_{2} x_{1}+z_{2} x_{2} y_{1}
\end{array}\right) .
$$

В алгебре $A$ рассмотрим систему подалгебр

$$
A \supseteq A^{[1]} \supseteq A^{[2]} \supseteq \cdots \supseteq A^{[\omega]} \supseteq A^{[\omega+1]} \supseteq \cdots,
$$

где $A^{[1]}=A^{3}, A^{[\alpha+1]}$ есть подалгебра, порожденная произведениями вида $a_{\alpha} u v$, $a_{\alpha} \in A^{[\alpha]}, u, v \in A$, a $A^{[\alpha]}$ с предельным $\alpha$ есть пересечение предыдущих. Если при некотором $\alpha A^{[\alpha]}=0$, то $A$ назовем ZD-алгеброй. Наименьшее $\alpha$, удовлетворяюшее условию $A^{[\alpha]}=0$, назовем ступенью $\mathrm{ZD}$-алгебры.

Алгебру $A$ будем назьвать правильной, если в $A$ существует базис $e_{\alpha}$, обладающий следующими свойствами:

1) произведение любых трех базисных элементов есть либо нуль, либо базисньй элемент;

2) если $a_{\alpha}, a_{\beta}$ - базисные элементы, то $a_{\alpha} a_{\beta}=-a_{\beta} a_{\alpha}$.

Базис с этими свойствами будем называть правильным. Легко убедиться, что правильная алгебра антикоммутативна.

Лемма 1. Ступень правильной ZD-алгебры $A$ совпадает со ступенью соответствующей ей коммутативной ZD-лупь Муфанг $Q$.

ДокАЗАТЕЛЬСтво. Из (2) и определения системы (3) следует, что если $A$ является $\mathrm{ZD}$-алгеброй, то KЛМ $Q$ является $\mathrm{ZD}$-лупой, и ступень $\mathrm{ZD}$-лупы $Q$ не выше ступени $\mathrm{ZD}$-алгебры $A$. Обратно, покажем, что все элементы базиса алгебры $A$, содержащиеся в $A^{[\alpha]}$, входят в $Q_{\alpha}$. При $\alpha=1$ это очевидно. Предположим, что утверждение справедливо для всех $\alpha$, меньших $\beta$. Если $\beta$ - предельное, то утверждение будет, очевидно, справедливьп. Поэтому предположим, что $\beta=\alpha+1$. Выберем произвольньй элемент базиса $e_{\alpha+1}$ из $A^{[\alpha+1]}$. Согласно определению алгебры $A^{[\alpha+1]}$ ее базис состоит из произведений вида $e_{\alpha} e_{1} e_{2}$, где $e_{\alpha} \in A^{[\alpha]}, e_{1}, e_{2} \in A$. По индукционному предположению

$$
\left(\begin{array}{c}
e_{\alpha} \\
e_{\alpha}
\end{array}\right) \in Q_{\alpha}
$$


Согласно (2) вычислим ассоциатор

$$
\left[\left(\begin{array}{c}
e_{\alpha} \\
e_{\alpha}
\end{array}\right),\left(\begin{array}{c}
e_{1} \\
0
\end{array}\right),\left(\begin{array}{c}
0 \\
e_{2}
\end{array}\right)\right]=\left(\begin{array}{c}
e_{\alpha} e_{1} e_{2} \\
e_{2} e_{\alpha} e_{1}
\end{array}\right)=\left(\begin{array}{c}
e_{\alpha} e_{1} e_{2} \\
e_{\alpha} e_{1} e_{2}
\end{array}\right) .
$$

Следовательно, ступень ZD-алгебры $A$ не превосходит ступени ZD-лупы $Q$. Лемма доказана.

Пусть заданы алгебры $A, B$ с базисами $e_{\alpha_{i}}, f_{\beta_{j}}(i, j=1,2, \ldots)$, и пусть $F$ - алгебра, порожденная множеством $\left\{e_{\alpha_{i}}, f_{\beta_{j}}: i, j=1,2, \ldots\right\}$ и удовлетворяюшая соотношению $e_{\alpha} f_{\beta}=-f_{\beta} e_{\alpha}$. Рассмотрим подалгебру $C$ алгебры $F$, составленную из всех элементов вида $e_{\alpha_{1}} e_{\alpha_{2}} f_{\beta}, e_{\alpha} f_{\beta_{1}} f_{\beta_{2}}, e_{\alpha}, f_{\beta}$. Назовем алгебру $C$ правильным произведением $A$ на $B$. Легко заметить, что если $A, B$ - правильные алгебры с правильными базисами $e_{\alpha}, f_{\beta}$, то правильное произведение $A$ на $B$ будет правильной алгеброй с правильным базисом $e_{\alpha_{1}} e_{\alpha_{2}} f_{\beta}, e_{\alpha} f_{\beta_{1}} f_{\beta_{2}}, e_{\alpha}, f_{\beta}$.

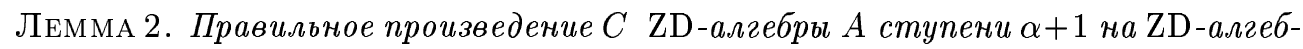
ру В ступени $\beta$ является ZD-алгеброй ступени $\geqslant \alpha+\beta$.

ДокАЗАтЕльство. Действительно, базис $C^{[\alpha]}$ содержит все элементы вида $e_{i} f_{j} f_{r}$, где $e_{i} \in A^{[\alpha]}, f_{j}, f_{r} \in B$. Отсюда видно, что базис $C^{[\alpha+\lambda]}(\lambda<\beta)$ содержит все элементы вида $e_{i} f_{j} f_{r}$, где $e_{i} \in A^{[\alpha]}, f_{j}, f_{r} \in B^{[\lambda]}$. Следовательно, $C^{[\alpha+\lambda]} \neq 0$, что и требовалось.

ЛЕмма 3. Для кажсдого трансфинитного числа а существуют правильные ZD-алгебры ступени $\alpha$.

ДокАЗАТЕЛЬСТво. Предположим, что $\alpha$ конечно. Пусть $B$ - внешняя алгебра, порожденная $2 \alpha+1$ элементами. Тогда в качестве искомой алгебры можно взять прямую сумму подалгебр $A^{2 k+1}$, где $k=1,2, \ldots$ Пусть теперь доказано, что правильные $\mathrm{ZD}$-алгебры ступеней, меньших $\alpha$, существуют. Если $\alpha$ предельное, то прямая сумма правильных ZD-алгебр ступеней, стремящихся к $\alpha$, даст правильную ZD-алгебру ступени $\alpha$. Пусть теперь $\alpha=\alpha^{\prime}+1, \alpha^{\prime}=\beta+1$. Тогда в силу леммы 2 произведение двух правильных ZD-алгебр ступеней $\beta+1$ даст правильную ZD-алгебру ступени $\beta+\beta \geqslant \alpha$. Осталось рассмотреть случай, когда $\alpha=\alpha^{\prime}+1$ и $\alpha^{\prime}$ предельное. Пусть $\alpha_{\lambda}<\alpha^{\prime}$, $\lim \alpha_{\lambda}=\alpha^{\prime}$. Для каждого $\lambda$ строим правильную ZD-алгебру $A_{\lambda}$ ступени $\alpha_{\lambda}+1$. Кроме того, мы можем считать, что размерности $\left(A_{\lambda}\right)^{\left[\alpha_{\lambda}\right]}$ при всех $\lambda$ одинаковы. Отобразим все $\left(A_{\lambda}\right)^{\left[\alpha_{\lambda}\right]}$ изоморфно на $\left(A_{\lambda}\right)^{\left[\alpha_{1}\right]}$. Теперь составим прямую сумму $A$ алгебр $A_{\lambda}$ и в этой сумме отождествим соответственные элементы подалгебр $\left(A_{\lambda}\right)^{\left[\alpha_{\lambda}\right]}$. В новой алгебре $\bar{A}$ все степени вида $\bar{A}^{\left[\alpha_{\lambda}\right]}$ содержат $\left(\bar{A}_{1}\right)^{\left[\alpha_{1}\right]}$ и, таким образом, $\bar{A}^{\left[\alpha^{\prime}\right]}$ также содержит $\left(A_{1}\right)^{\left[\alpha_{1}\right]}$. Отсюда видно, что ступень $\mathrm{ZD}$-алгебры $\bar{A}$ равна требуемому числу $\alpha^{\prime}+1$. Лемма доказана.

ТЕорема 1. Для каждого трансфинитного числа а существует коммутативная лупа Муфанг, длина нижнего чентрального ряда которой равна $\alpha$.

Утверждение следует из лемм 1,3 .

Пусть теперь $A$ - алгебра с базисом $e_{\alpha \beta}$, где $\alpha, \beta$ пробегают множество трансфинитных чисел $1,2, \ldots, \omega, \omega+1, \ldots, \alpha<\beta$, и таблицей умножения $e_{\alpha \beta} e_{\beta \gamma}=-e_{\beta \gamma} e_{\alpha \beta}$, $e_{\alpha \beta} e_{\beta \gamma} e_{\gamma \delta}=e_{\alpha \delta}$. Обозначим через $Q$ КЛМ, соответствующую алгебре $A$, и пусть

$$
Q \supseteq Q^{(1)} \supseteq Q^{(2)} \supseteq \cdots \supseteq Q^{(\omega)} \supseteq Q^{(\omega+1)} \supseteq \cdots
$$


- производньй ряд ряд лупы $Q$. Введем функцию $\varphi(\alpha)$ с помощью соотношений

$$
\varphi(0)=1, \quad \varphi(\alpha+1)=\varphi(\alpha)+\varphi(\alpha)+\varphi(\alpha), \quad \varphi(\beta)=\lim _{\lambda<\beta} \varphi(\lambda)
$$

где $\beta$ предельное. Утверждается, что $Q^{(\lambda)}$ содержит все элементы вида

$$
\left(\begin{array}{c}
e_{\alpha \beta} \\
0
\end{array}\right), \quad\left(\begin{array}{c}
0 \\
e_{\alpha \beta}
\end{array}\right),
$$

у которых $\beta \geqslant \alpha+\varphi(\lambda)$. Действительно, для $\lambda=0$ это тривиально, так как по определению $Q^{(0)}=Q$. Допустим, что утверждение верно для всех значений $\lambda$, меньших некоторого $\sigma$. Если $\lambda$ предельное, то утверждение будет, очевидно, справедливым и для $\sigma$. Осталось рассмотреть случай $\sigma=\rho+1$. Возьмем некоторый элемент $e_{\alpha \delta}, \delta \geqslant \alpha+\varphi(\sigma)$. Этот элемент можно представить в виде $e_{\alpha \delta}=e_{\alpha \beta} e_{\beta \gamma} e_{\gamma \delta}$, где $\beta=\alpha+\varphi(\rho), \gamma=\beta+\varphi(\rho), \delta \geqslant \gamma+\varphi(\rho)$. Тогда $\gamma \geqslant \alpha+3 \varphi(\rho)=\alpha+\varphi(\rho+1)$. По условию

$$
\left(\begin{array}{c}
e_{\alpha \beta} \\
0
\end{array}\right), \quad\left(\begin{array}{c}
e_{\beta \gamma} \\
0
\end{array}\right), \quad\left(\begin{array}{c}
0 \\
e_{\gamma \delta}
\end{array}\right)
$$

входят в $Q^{(\rho)}$. Согласно $(2)$

$$
\left[\left(\begin{array}{c}
e_{\alpha \beta} \\
0
\end{array}\right),\left(\begin{array}{c}
e_{\beta \gamma} \\
0
\end{array}\right),\left(\begin{array}{c}
0 \\
e_{\gamma \delta}
\end{array}\right)\right]=\left(\begin{array}{c}
e_{\alpha \beta} e_{\beta \gamma} e_{\gamma \delta} \\
0
\end{array}\right)=\left(\begin{array}{c}
e_{\alpha \delta} \\
0
\end{array}\right) .
$$

Но $\gamma \geqslant \alpha+\varphi(\rho+1)$, откуда

$$
\left(\begin{array}{c}
e_{\alpha \delta} \\
0
\end{array}\right) \in Q^{(\sigma+1)}
$$

Таким образом, выбирая достаточно высокие значения для $\sigma$, получаем следующее утверждение.

ТЕОРема 2. Для каждого трансфинитного числа а существует коммутативная лупа Муфанг, длина производного ряда которой равна $\alpha$.

Наконец, из доказательства теоремы 1 следует

TЕорема 3. Коммутативные RK-лупы Муфанг ступени $\omega$ могут быть ZD-лупами произвольных ступеней.

\section{СПИСОК ЦИТИРОВАННОЙ ЛИТЕРАТУРЫ}

1. Bruck R. H. A survey of binary systems. Berlin: Springer Verlag, 1958. 2. Malbos C. P. // C. R. Acad. Sci. Paris. Ser. A. 1978. V. 287. №9. P. 691-693. 3. Beneteau L. // J. Algebra. 1980. V. 67. Р. 1-35. 4. Мальцев А. И. // Матем. сб. 1949. Т. 25. №3. С. 347-366. 